\title{
Erratum to: The isolation and characterization of 10 dinucleotide microsatellite markers from enriched Channa argus genomic library
}

\author{
Yasmeen Gul • Weimin Wang · Huanling Wang
}

Published online: 11 March 2010

(C) Springer Science+Business Media B.V. 2010

\section{Erratum to: Conservation Genet Resour}

DOI 10.1007/s12686-009-9163-z

The paper contains three errors where the word/number eleven/11 should be corrected as ten/10:
Abstract, first paragraph
Eleven microsatellite markers were isolated... should be
Ten microsatellite markers were isolated...

\section{Main text, third paragraph}

Annealing temperatures were optimized for these 11 loci using...

should be

Annealing temperatures were optimized for these 10 loci using...

The last 11 primers produced DNA products (Table 1). should be

The last 10 primers produced DNA products (Table 1).

The online version of the original article can be found under doi: 10.1007/s12686-009-9163-z.

Y. Gul $\cdot$ W. Wang $(\bowtie) \cdot H$. Wang

College of Fisheries, Key Lab of Freshwater Biodiversity Conservation and Utilization, Ministry of Agriculture, Key Lab of Agricultural Animal Genetics, Breeding and Reproduction of Ministry of Education, Huazhong Agricultural University, 430070 Wuhan, China

e-mail: wangwm@mail.hzau.edu.cn 